\title{
Concomitant Rupture of Extensor Pollicis Longus and Index Extensor Tendons following Distal Radius Plating-Report of Two Cases and Their Clinical Implication for Tendon Transfer Surgery
}

\author{
Praveen Bhardwaj' Vigneswaran Varadharajan² \\ ${ }^{1}$ Department of Hand, Wrist Surgery and Reconstructive \\ Microsurgery, Ganga Hospital, Coimbatore, Tamil Nadu, India \\ 2Department of Plastic, Hand and Reconstructive Microsurgery, \\ Ganga Hospital, Coimbatore, Tamilnadu, Indiaa \\ ${ }^{3}$ Department of Plastic, Hand, Burns and Reconstructive \\ Microsurgery, Ganga Hospital, Coimbatore, Tamil Nadu, India
}

\author{
S Raja Sabapathy ${ }^{3}$
}

Indian J Plast Surg:2020;53:435-438

\begin{abstract}
Address for correspondence Praveen Bhardwaj, MS, DNB (Ortho); FNB (Hand \& Microsurgery), EDHS, Department of Hand, Wrist Surgery and Reconstructive Microsurgery, Ganga Hospital, Coimbatore, Tamil Nadu, India (e-mail: drpb23@gmail.com).
\end{abstract}

\begin{abstract}
Keywords

- rupture of extensor pollicis longus

- extensor indicis proprius

- distal radius fracture

- extensor tendon rupture

Extensor indicis proprius (EIP) tendon transfer is a standard operation for restoration of the thumb extension following rupture of extensor pollicis longus (EPL). In its standard form often the EIP is transferred to the EPL without inspection of the extensor tendons in the fourth compartment and it is retained in its anatomical fourth compartment. However, in a setting of EPL rupture in relation to the distal radius fracture (with or without fixation), concomitant injury to the extensor tendons to the index finger may result in failure of the transfer and even a loss of index finger extension (index finger drop) further complicating the reconstruction and resulting in immense patient dissatisfaction. We herein present two such rare cases to highlight this clinical scenario and how an awareness of this possibility and inspection of the extensor tendons to the index finger before EIP transfer allowed us to prevent this complication. In essence, if we know it, we can prevent it.
\end{abstract}

\section{Introduction}

Extensor indicis proprius (EIP) to extensor pollicis longus (EPL) transfer is the most commonly used option for the restoration of thumb extension following rupture or injury to the EPL and is considered reliable. ${ }^{1}$ It is technically easy and its excursion and approach from the ulnar side resemble the EPL rendering it a good replacement. Moreover, its removal does not cause any appreciable functional deficit and even the independent extension of the index finger is retained in most of the patients. ${ }^{1,2}$ This transfer probably is the first choice of many surgeons, including us, for EPL rupture because of the close proximity of the donor and its direct reach to the EPL without any intervening graft. However, a concomitant injury to the extensor digitorum (ED) of the index finger in cases of EPL rupture in relation to the distal radius fracture may result in a patient losing extension at the index finger (index drop) after EIP transfer. Unfortunately, it is not possible to clinically identify the function of ED if the EIP is working making this a potentially serious complication of EIP transfer in this particular situation. Concomitant rupture of the EPL and ED of the index is fortunately rare; however, the serious implication needs a wider awareness of this possible complication. ${ }^{3-5}$ We herein report two cases highlighting this rare but disastrous complication that merits a serious consideration while contemplating EIP transfer for thumb extension in distal radius fracture-related ruptures of the EPL. published online

December 25, 2020
DOI https://doi.org/

10.1055/s-0040-1721859 ISSN 0970-0358.
C2020. Association of Plastic Surgeons of India.

This is an open access article published by Thieme under the terms of the Creative Commons Attribution-NonDerivative-NonCommercial-License, permitting copying and reproduction so long as the original work is given appropriate credit. Contents may not be used for commercial purposes, or adapted, remixed, transformed or built upon. (https://creativecommons.org/licenses/by-nc-nd/4.0/).

Thieme Medical and Scientific Publishers Pvt. Ltd. A-12, 2nd Floor, Sector 2, Noida-201301 UP, India 


\section{Case Reports}

\section{Case 1}

A 69-year-old female, right hand dominant, who was asymptomatic for 18 years following internal fixation of left distal radius fracture noted inability to extend the thumb. She was diagnosed with EPL rupture of the left thumb elsewhere and underwent implant removal and EIP transfer to the EPL. Two years later she presented to us with inability to extend the left thumb and index finger. She reported that the loss of index finger extension is present since the tendon transfer operation, while the thumb dropped a few months after the tendon transfer surgery. She has not considered medical consultation as she was not satisfied with the previous operation but reported functional disability. There were no associated comorbid conditions, such as rheumatoid arthritis, which could account for tendon rupture. Ultrasound examination of the wrist revealed discontinuity of the extensor tendons to the index finger at the wrist, while the other extensor tendons were intact. On exploration, the EIP-EPL transfer was found ruptured and the extensor digitorum communis (EDC) tendon to the index finger, as well, was ruptured (-Fig. 1). The patient underwent side-hitching of the index finger EDC to middle finger EDC and palmaris longus to EPL transfer with hemi-flexor carpi radialis graft. She achieved good thumb and index extension after the surgery and had good functional outcome and was satisfied with the operation.

\section{Case 2}

A 64-year-old female, right hand dominant, who underwent fixation of bilateral distal radius fracture with volar locking compression plate 6 years ago presented with sudden drop of left thumb for 20 days (-Fig. 2). The right side was asymptomatic. There were no associated comorbid conditions, such as rheumatoid arthritis, which could account for tendon rupture. On examination of the left hand, there was absent thumb extension and retropulsion with intact independent extension of the index finger. Radiographs showed satisfactory union and no other investigation was performed (-Fig. 3). On exploration, there was a prominent screw found protruding beyond the dorsal cortex in the fourth extensor compartment, with ruptured EDC tendon to index finger (-Figs. 4 and 5). The EPL tendon was also found ruptured, the reason for which was not clear as there was no screw prominence in the third compartment. However, it could have been because of the attrition against the rough bone fracture edges, or drilling while surgery or compartment syndrome of the EPL tendon or a combination of these factors. The implant was removed and the EPL tendon was reconstructed with intertendinous grafting using ipsilateral palmaris longus graft. Thumb extension till neutral was possible and it could easily reach all the fingers with flexion without any tightness. Patient had good improvement in thumb extension and was satisfied with the outcome of surgery (-Fig. 6).
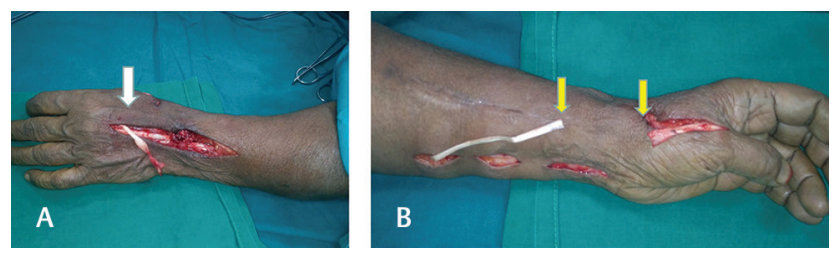

Fig. 1 Intraoperative pictures of case 1, showing (A) the ruptured extensor digitorum slip to the index finger (arrow). It was side-hitched to the middle finger slip of the extensor digitorum. (B) Distal segment of the ruptured extensor pollicis longus (distal arrow). Palmaris longus (proximal arrow) extended with hemi-flexor carpi radialis tendon graft was transferred to the extensor pollicis longus for thumb extension.

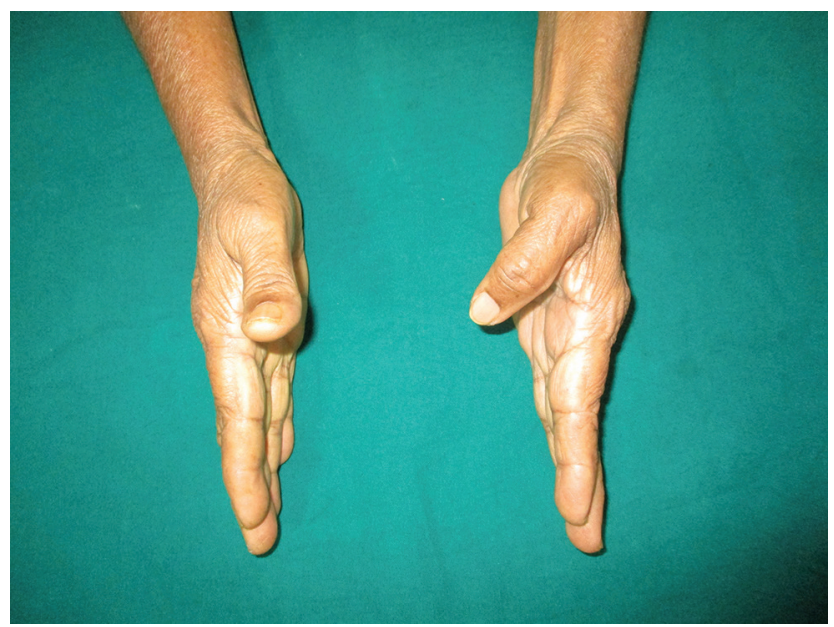

Fig. 2 Preoperative picture showing the drop of the thumb on the left side.

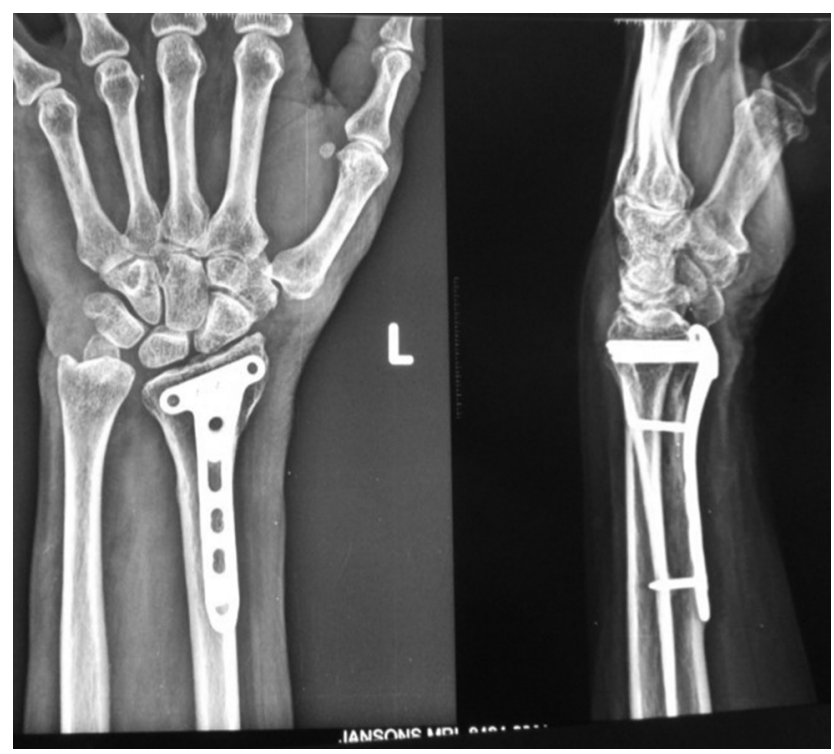

Fig. 3 Preoperative X-ray showing well-healed fracture with reasonable length of screws and no obviously long screw. The Lister's tubercle probably obscures the actual length of the screws. 


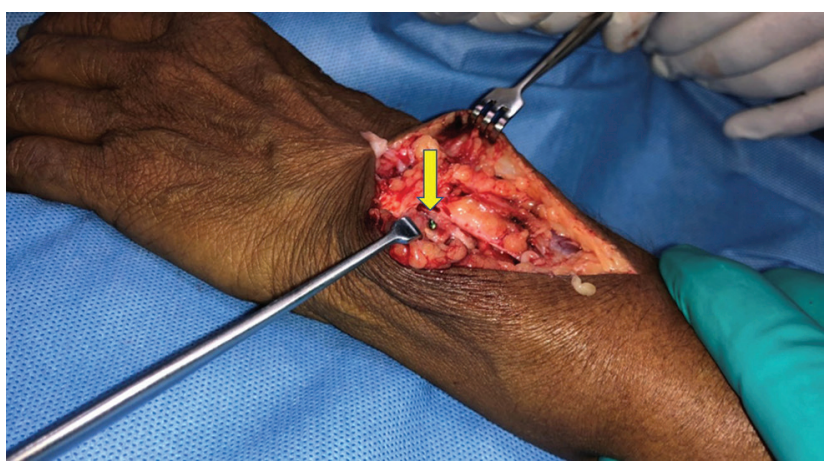

Fig. 4 Intraoperative photos showing the prominent screw tip in the fourth compartment just next to the Lister's tubercle that caused the attrition rupture of the extensor digitorum slip to the index finger.

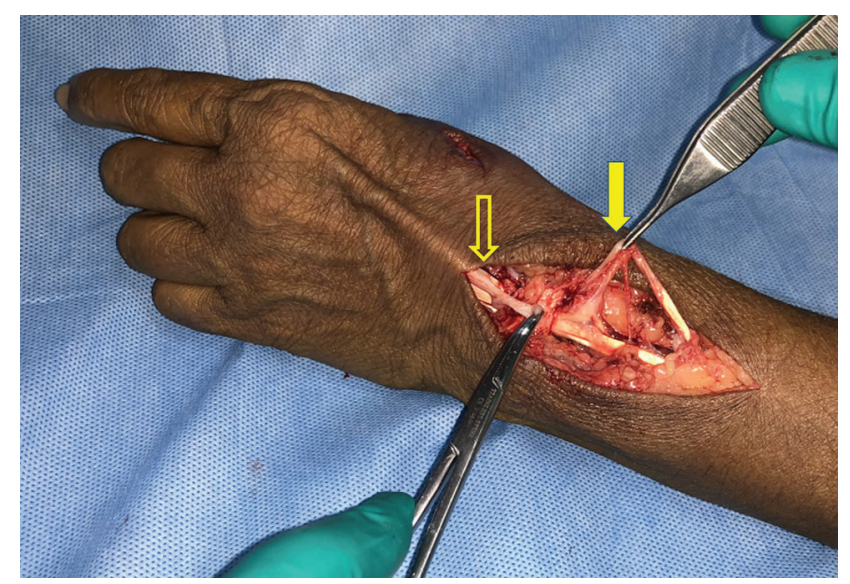

Fig. 5 Intraoperative picture showing the ruptured extensor digitorum slip of the index finger (open arrow) held in the hemostat and proximal cut end of the ruptured extensor pollicis longus (arrow) stuck in the scar tissue over the third compartment.

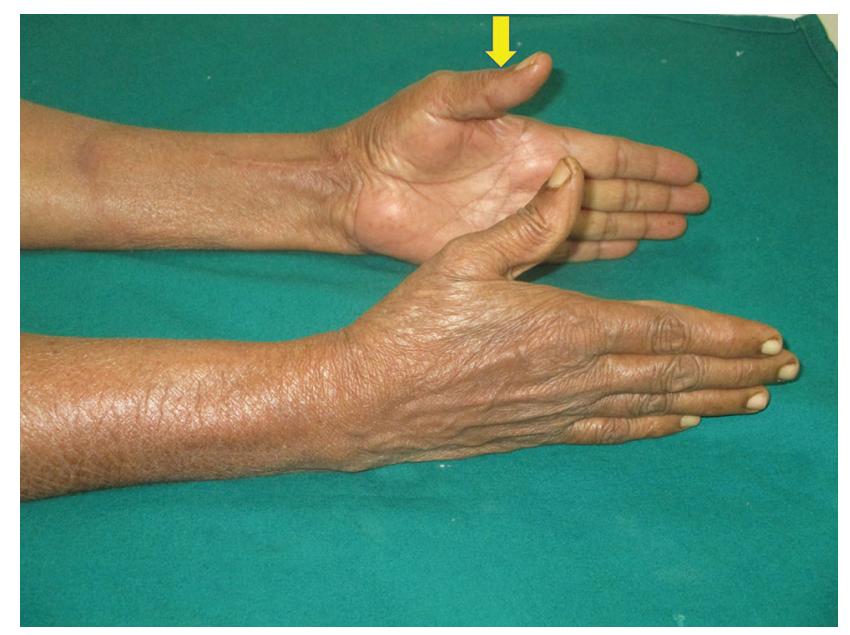

Fig. 6 Postoperative outcome was satisfactory with good thumb extension.

\section{Discussion}

The reported incidence of EPL rupture in relation to the distal radius fracture is variable. The implant-related rupture rates have reduced with better designs and preventive measures adopted by the surgeons. Recent publications cite an incidence with volar plating as $0.8 \%$ and those with dorsal plating as $0.3 \% .{ }^{6}$ However, the EPL ruptures can happen even with undisplaced distal radius fractures. ${ }^{7}$ The causes for the EPL ruptures are injury by the penetrating drill bit, projecting screw tip dorsally, attrition from the bone ridge or callus or the plate, and compromised vascularity of the EPL tendon because of increased pressure in the third compartment in cases of undisplaced distal radius fractures..$^{2-7}$ Injury to the other extensor tendons has also been reported..$^{3-6,8}$

The ED slip of the index finger is the next adjacent tendon to the EPL and the projecting screw or the over penetrating drill or rough bony e.g. around the Lister's tubercle could as well cause damage to this tendon. Strangely enough, it is not possible to detect the isolated injury to ED slip to index finger clinically as the patient would exhibit good independent index extension by virtue of the intact extensor indicis, as was possible in our case 2 . In such a situation, the standard practice of transfer, by transferring the EIP to the EPL without inspecting the fourth compartment and leaving the EIP in the fourth compartment, would leave no extensor for the index finger resulting in index drop. Even concerning is the fact that if the transfer has been done without removing the EIP from the fourth compartment the EIP would now be at the location of the ED and would be exposed to the factors that resulted in the ED rupture and could have an attrition rupture in the future as has happened in our case 1 and reported by Heo et al in their interesting case report. ${ }^{5}$

We recommend inspection of the EIP and ED slip of index finger to confirm their intactness and rule out any attrition with partial injury for the possibility of concomitant injury and transfer of the EIP out of the fourth compartment while performing EIP to EPL transfer in distal radius fracture-related ruptures. The EIP and ED tendon to index can be pulled proximally to move the index finger to confirm their continuity. Also, a proximal and distal pull on the tendon across the retinaculum can allow visualization of the part under the retinaculum to rule out any partial attrition injury. If a partial attrition injury is noted, or if there is a strong suspicion for attrition damage, then the retinaculum could be partly opened to inspect for any projecting screw or sharp bony prominence. Assessment of the integrity of the index extensor tendons by pulling distally alone could be misleading in cases of attrition damage with partial injury because the attrition happens over a period of long time and even if few tendon fibers are intact, the tendon would move on pulling and could be wrongly judged as "Intact"; only to rupture during rehabilitation, if this tendon was used for transfer. If index extensors are found injured, an alternative motor (e.g. palmaris longus, brachioradialis, extensor carpi radialis longus, flexor digitorum superficialis) should be kept in mind.

\section{Conclusion}

The EIP to EPL transfer could indeed remain the preferred transfer for thumb extension for restoring thumb extension in cases of EPL rupture; however, when dealing with distal 
radius fracture-related cases one must explore to find out that the ED slip to the index is uninjured and functioning fine before embarking on the EIP transfer to EPL and leave the transfer outside the compartment in the subcutaneous plane. This would avoid a rare but disastrous situation of ending up with index finger drop and prevent rerupture of the transfer. In case, if one finds an associated injury to the ED slips of index an alternative option of restoring thumb extension should be used.

\section{Conflict of Interest}

None declared.

\section{References}

1 Magnussen PA, Harvey FJ, Tonkin MA. Extensor indicis proprius transfer for rupture of the extensor pollicis longus tendon. J Bone Joint Surg Br 1990;72(5):881-883

2 Moore JR, Weiland AJ, Valdata L. Independent index extension after extensor indicis proprius transfer. J Hand Surg Am 1987;12(2):232-236
3 Sadr B. Sequential rupture of extensor tendons after a Colles fracture. J Hand Surg Am 1984;9A(1):144-145

4 de Boer SW, van Kooten EO, Ritt MJ. Extensor pollicis longus tendon rupture with concomitant rupture of the extensor digitorum communis II tendon after distal radius fracture. J Hand Surg Eur Vol 2010;35(8):679-681

5 Heo YM, Won Y, Lee YM, Kim TG, Lee JIK. Failed extensor indicis proprius tendon transfer for extensor pollicis longus tendon rupture after distal radial fracture. J Korean Soc Surg Hand 2015;20:23-27

6 Azzi AJ, Aldekhayel S, Boehm KS, Zadeh T. Tendon rupture and tenosynovitis following internal fixation of distal radius fractures: a systematic review. Plast Reconstr Surg 2017;139(3): 717e-724e

7 Hirasawa Y, Katsumi Y, Akiyoshi T, Tamai K, Tokioka T. Clinical and microangiographic studies on rupture of the E.P.L. tendon after distal radial fractures. J Hand Surg [Br] 1990;15(1):51-57

8 Singh HP, Srinivasan S, Ullah A. Closed rupture of the extensor indicis and extensor digitorum tendons to the index finger after locking plate fixation of a fracture of the distal radius. J Hand Surg Eur Vol 2013;38(1):86-87 